\title{
Pavel Salák
}

Uniwersytet Masaryka w Brnie e-mail: 10908@mail.muni.cz

\section{Die Rechtsregelung von Straßenalleen vom 18. bis ins 21. Jahrhundert in böhmischen Ländern}

\section{SUMMARY \\ The Legal Regulation of the Alleys of Trees along the Roads on Czech Territory from 18th to 21 st Century}

The temperate winter of 2006/2007 prompted the road maintenance board in some places within the Czech Republic to cut down the alleys of trees along the roads. These alleys started in 18th century, but were planted mostly in the first half of the 19th century, when the roads were built on a massive scale.

Of course, together with the regulations of these roads, the planting of these alleys was also regulated by rules. Not only was the distance of the trees from the road regulated, but also the sort of trees that were planted, depending upon the climate conditions in the given locality. Because they shared the costs of planting, owners of the grounds preferred fruit trees. There were also penalties assessed for any damage done to the trees. To plant the alleys faster, school children were engaged in the growing of young trees. So from the 19th to the end of the 20th century, the alleys were a distinctive part of the Czech landscape.

With the massive increase of traffic, alleys were increasingly threatened. First, the expansion of roads combined with increasing vehicle speed caused such alleys to become regarded as dangerous obstacles. Indeed, traffic safety was the main argument for the removal of trees along the roads. However, after the alleys began to be cut during the winter of 2006/2007, several weeks later the nature protection office and the traffic office stopped the cutting. Today, as well as in the past, the alleys have been important not only as a part of the landscape,

*_ JUDr. Pavel Salák, Ph.D., Lehrstuhl für Rechts- und Staatsgeschichte, Juristische Fakultät der Masaryk - Universität Brno, Tschechische Republik. 
but also for the traffic. Some studies from Austria can show, that cutting of alleys has led to an increase of the quantity of accidents caused by high speed - the drivers felt overconfident and thus and drove too fast.

Key words: alleys of trees, Habsburg monarchy, administration, Czech territories

In der Tschechischen Republik kommt es zu vielen Verkehrsunfällen mit Todesfolgen oder schweren Verletzungen durch Baum-Aufprall. Im Jahre 2006 endeten 149 dieser Unfälle tödlich. Das sind $15 \%$ aller tödlichen Unfälle auf tschechischen Straßen. Es scheint also eine ziemlich hohe Zahl zu sein, andererseits stellte die Unfallursache in der Regel kein Baum, sondern in den meisten Fällen eine rücksichtslose Fahrt dar ${ }^{1}$. Kein Wunder, dass es oft Stimmen seitens der Öffentlichkeit für die Abschaffung dieser Bäume gibt. Ein milder Winter 2006/2007 brachte für ein solches Fällen eine ideale Gelegenheit, die auch in einer Reihe von Ortschaften genutzt wurde. Die Medien berichteten damals vor allem über das Fällen von Alleebäumen bei der Gemeinde Pohled im Jahr 2007. Die Autobahnen- und Straßenverwaltung ließ hier eine Allee von angestammten Kastanien fällen².

Die Bedeutung von Baumalleen längs der Straßen charakterisierte der Erlass des Ministeriums für öffentliche Arbeiten vom 9.12.1924, Az. 11 b-96/5-1923/8868-1924 sehr gut. Nach diesem Erlass ist eine Baumallee:

- ein wichtiges Orientierungsmittel - vor allem in der Nacht, im Winter oder im Falle des Hochwassers;

- sie verhindert den eventuellen Autosturz in den Teich oder in die Schlucht;

- sie verfügt über eine ästhetische Ausprägung und trägt zu einer schönen Landschaft bei;

- schließlich ist es eine Einkommensquelle - was vor allem Obst betrifft. ${ }^{3}$

V. Ševela, Stačí zpomalit, In: Respekt, 26. Februar 2007.

2 Hier ist auch eine Forderung der EU zu erwähnen: sie verlangt jedoch nicht das Baumfällen, sondern nur die Senkung der Zahl von Verkehrsunfällen auf den Straßen. Dazu vgl. V. Ševela, Stačí zpomalit, In: Respekt, 26. Februar 2007.

3 Zitiert nach: Slovník veřejného práva československého (Schlagwort „Silnice a cesty veřejné“, bearbeitet von: Jiří Čistecký). IV. Band. Praha: Eurolex Bohemia, 2000 (Reprint), S. 221. 


\section{Anfänge der Rechtsregelung (18. bis erste Hälfte des 19. Jahrhunderts)}

Die Geschichte der Baumalleen längs der Wege ist fast so alt, wie die Wege selbst. Trotz der Tatsache, dass sich die Bäume längs der Wege schon seit uralten Zeiten befanden, finden wir eine entsprechende Rechtsregelung erst im 18. Jahrhundert unter der Herrschaft von Maria Theresia. Dass ist kein Zufall; diese Tatsache wurde von zwei Faktoren beeinflusst.

$\mathrm{Zu}$ dieser Zeit wurde mit dem Aufbau eines modernen Straßennetzes begonnen (die sog. Kaiserstraßen). Nach dem franzsischen Vorbild sollten sie die Verkehrsachsen darstellen und bedeutende strategische und Handelsortschaften verbinden. Bis Ende des 18. Jahrhunderts wurden nur etwa 1400 Kilometer Straßen in Böhmen gebaut, wobei der Straßenbau in Mähren und in Schlesien langsamer verlief. Aufgrund einer schnellen Wirtschaftsentwicklung und einem steigendenden Umfang der beförderten Ware steigerten sich die Aktivitäten im Straßenbau. Bis 1850 war das Straßennetz in Böhmen und Mähren praktisch vollendet, wobei es ca. 4000 Kilometer aufwies ${ }^{4}$.

Einen anderen Faktor stellt die Tatsache dar, dass es ein typisches Bemühen für die Zeit der Aufklärung ist, die Gesellschaftsverhältnisse oft bis in lächerliche Details zu regeln. Es ist also kein Wunder, dass wir neben den den Straßenaufbau betreffenden Vorschriften auch solche Vorschriften finden, welche die Anpflanzung von Bäumen längs dieser Wege regeln.

Die erste Vorschrift stellte das Allerhöchste Reskript vom 21.3.1740 dar. Ein weiteres folgte am 29.08.1752 und schließlich am 24.09.17635. Diese Vorschriften empfahlen jedoch nur eine Anpflanzung von Bäumen und führten nur Beispiele der in Betracht kommenden Baumarten an, die angepflanzt werden sollten. Diese Anpflanzung, sowie der Straßenbau gehörten in die Zuständigkeit der einzelnen Dominien, Gemeinden und Städte.

Von der Tatsache, dass die Obrigkeit dies oft nicht getan hat, zeugt auch die Allerhöchste Entschließung vom 29. Januar 1780, die auch noch als Hofdekret am 12.05.1781 veröffentlicht wurde. $\mathrm{Zu}$ einer Wiederveröffentlichung kam es noch einmal am 7.12.1781. Hier war nicht nur die Anordnung enthalten, die

4 Obrázky z dějin poštovnictví XIV. - Na cestách s poštou, http://www.infofila.cz/new/cteni.php?r $=2 \& c=1421$, zitiert am 10. Dezember 2007, 16 Uhr.

5 Das Letztgenannte wurde veröffentlicht in: Sammlung aller k. k. Verordnungen und Gesetze vom Jahre 1740 bis 1780, die unter Regierung des Kaisers Joseph des II. theils nochganz bestehen, theils zum Theile abgeändert sind, als ein Hilfs- und Ergänzungsbuch zu dem Handbuche aller unter der Regierung des Kaisers Joseph des II. für die k. k. Erbländer ergangenen Verordnungen und Gesetze in einer chronologischen Ordnung. IV. Band. Wien: Joh. Georg Mössle k.k. prov. Buchhändler, 1786. Zugänglich unter: http://alex.onb.ac.at/cgi-content/alex?aid=tgb\&datum $=1766 \&$ page $=278 \&$ size $=45$, zitiert am 10. Januar 2012, $10 \mathrm{Uhr}$. 
Bäume anzupflanzen, sondern es waren auch weitere Einzelheiten festgelegt. Die Bäume sollten auf der Außenseite des Straßengrabens angepflanzt werden, damit sie die Feuchte ziehen konnten und damit die Wurzeln die Straßenfläche nicht bedrohten. Neben dieser technischen Anforderung führte das Dekret auch eine ästhetische an. Es machte darauf aufmerksam, dass auch die Gleichförmigkeit bei der Anpflanzung von Bäumen beachtet werden sollte, „weil es sonst dem Auge unangenehm fallen müsste, auf zweien nebeneinander liegenden Feldern

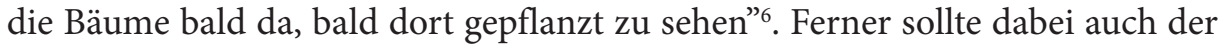
weitere Bedacht in der nachfolgenden Pflege nach der Anpflanzung genommen werden. Die Bäume sollten lediglich abgeästet, nicht aber geköpft werden. Der Baum gehörte selbstverständlich dem Grundbesitzer; er durfte den Baum nur schlagen, falls dieser abstarb. Der letzte Absatz dieses Dekrets betraf die Strafen - für jeden beschädigten Baum sollten 2 Fl. bezahlt werden.

Die Anpflanzung der Bäume und ihre Pflege wurden also den Grundbesitzern anvertraut, deren Grundstücke an den Straßen grenzten. Sie fanden für diese Arbeit jedoch kein großes Verständnis. Es wurden nicht so viele Bäume längs der Straßen angepflanzt, wie es sich die Staatsorgane vorgestellt hätten. Nachdem sich die mit einer Wirtschaftskrise teilweise verbundene Situation nach den napoleonischen Kriegen beruhigte, begannen sich die Behörden mit den Alleen wiederum zu beschäftigen. Im Sommer 1828 wurde eine neue sehr umfangreiche Vorschrift erlassen 7 . Diese Vorschrift versuchte schon in ihrem ersten Paragrafen einen größeren Eifer zur Bäumeanpflanzung anzuregen, indem sie die Vorteile für den Grundbesitzer anführte. Angesichts der Tatsache, dass der Eigentümer den Baum erst nach seinem Absterben schlagen durfte, was keinen großen Profit bedeutete, empfahl das Dekret vor allem die Anpflanzung von Obstbäumen. Auch diese Vorschrift erreichte jedoch offensichtlich ihr Ziel nicht, sodass eine Novellierung (Gub. Präsidialverordnung Gub. Präs. Z. 2097 vom 15.5.1831) $)^{8}$ erlassen wurde. $\mathrm{Zu}$ den größeren Übertretungen gehörten die Fälle, wenn die Bäume auf einem Grundstück angepflanzt wurden, während es auf dem anderen Grundstück nicht der Fall war, oder wenn die Bäume ohne Zustimmung der entsprechenden Straßenbehörde ausgewählt wurden, oder wenn nur eine bloße Rute statt der Bäume mit einer hohen Krone angepflanzt wurde. Es ist auch sehr interessant, den Zweck der Anpflanzung von Alleen zu verfolgen. Obwohl ich am Anfang dieser Abhandlung eine Reihe von Gründen erwähnte, warum eine Allee längs dem Wege vorteilhaft ist, führte keine dieser

6 Hofentschliessung von 28. Jänner 1780. Handbuch aller unter der Regierung des Kaisers Joseph des II. für die K.K. Erbländer ergangenen Verordnungen und Gesetze in einer Systematischen Verbindung 1780-1784. Band 3, Wien 1785, S. 504.

7 Gubernialverordnung vom 17. Juli 1828 Gub. Zahl. 17167, an die k. Kreisämter und die Straßenbaudirection. Provinzial-Gesetzsammlung des Königreichs Böhmen für das Jahr 1828, S. 270-278.

8 Provinzial-Gesetzsammlung des Königreichs Böhmen für das Jahr 1831, S. 228. 
Vorschriften einen solchen Grund an. Die Rede war immer nur von einer Verschönerung der Landschaft ${ }^{9}$.

Die Verordnung von 1828 beschrieb den Anpflanzungsprozess ausführlich. Wie schon oben erwähnt wurde, sollte es sich um nur eine Baumart auf einem Gebiet handeln. Die Baumart wurde vom entsprechenden Straßenkommissariat und örtlichen Behörden festgesetzt, ggf. entschied darüber die Kreisbehörde. Zu den Hauptbaumarten gehörten vor allem Birnen, Apfelbäume, Kirschen und Pflaumen, weiter Ahorne und Eichen. Für das Hochland wurden Vogelbeeren und Vogelkirschen bestimmt; im Tiefland war auch Pappel zulässig. Die Auswahl geschah entspechend dem Untergrunds und der Meereshöhe. Im Falle, dass schon einige Bäume längs der Straße angepflanzt wurden, mussten auch gleichartige Bäume angepflanzt werden. Es wurde auch eine Distanz zwischen den einzelnen Bäumen auf jeder Seite festgesetzt, wobei die Bäume abwechselnd auf beiden Seiten angepflanzt wurden ${ }^{10}$. Diese Vorschrift legte nicht nur die Größe der Grube zum Anpflanzen, sondern auch die Zeit fest, wann die Bäume gefällt werden sollten, die als Baumpfahl dienen sollten. Die Verordnung ordnete sogar an, dass es notwendig war, den Stock auf dem Unterstützungspunkt mit Stroh oder Moos umzuwickeln, damit es zu keiner Beschädigung kam ( $\$ 12)$. Der Grund für eine so detaillierte Regelung lag darin, dass diese Vorschrift auch als eine Anleitung zum Anpflanzen von Bäumen dienen sollte, denn dies führten einfache Leute durch, welche mit dem Obstbau nicht vertraut waren. Über die Tatsache, dass der Schaden auf der angepflanzten Allee oft durch fehlerhaften Fortgang verursacht wurde, zeugt auch der Bericht des Prager Straßenkommissariats vom 11. April 1833. Aus diesem Grunde wurde daher ein noch viel ausführlicherer Ablauf erlassen ${ }^{11}$.

Das Landespräsidium hat einen Beschluss gefasst, dass auch Schulen in die Anpflanzung von Bäumen einbezogen werden. Deswegen wurde das Fach "Anpflanzung von Bäumen und Obstbau“ in die Schulpläne an den Hauptund Trivialschulen vorgeschlagen ${ }^{12}$. Für uns sind die Gründe des Guberniums

9 „Da diese Vorschrift den Zweck hat, dem Lande Böhmen durch Verschönerung der Kunststraßen eine neue Zierde...” \$23 Gubernialverordnung vom 17. Juli 1828 Gub. Zahl. 17167. Provinzial-Gesetzsammlung des Königreichs Böhmen für das Jahr 1828, S. 277.

10 Der Abstand zwischen den Obstbäumen betrug 5 Kurrentklafter auf einer Seite, zwischen den Wildbäumen 6 Klafter. Dazu siehe $₫ 6$ Gubernialverordnung vom 17. Juli 1828 Gub. Zahl. 17167. Provinzial-Gesetzsammlung des Königreichs Böhmen für das Jahr 1828, S. 27). Ein Klafter beträgt ca. 1,896 m. Vgl. Vademecum pomocných věd historických. Praha: Svoboda, 1988, Tabelle IV, S. 169.

11 Setzungsmethode der Alleebaumpflanzungen und Erneuerung diessfalls bereits bestehenden Vorschriften. Gub. Veroderung vom 7. Oktober G. Z. 40493, an die k. Kreisämter und die k.k. Oberbaudirecion. Provinzial-Gesetzsammlung des Königreichs Böhmen für das Jahr 1833, S. 535-536.

12 Gubernialverordnung vom 1. Mai 1829 G.Z. 18253. Provinzial-Gesetzsammlung des Königreichs Böhmen für das Jahr 1829, S. 157-161. 
interessant und vielleicht auch aufschlussreich. Einerseits konnten die Kinder in der Schule über die Zweckmäßigkeit der Anpflanzung von Bäumen einfacher überzeugt werden, als ihre Eltern, andererseits bestand die Bedeutung auch darin, dass die Kinder, die von Jung auf für die Bäume Sorge tragen, zu diesen auch ein anderes Verhältnis haben. Sie werden also die Bäume schützen und nicht beschädigen und diese Einstellung übertragen sie auch auf ihre Kinder ${ }^{13}$.

Wie schon oben erwähnt wurde, für die Beschädigung von Bäumen wurden verhältnismäßig hohe Finanzstrafen auferlegt. Zur Hebung der Motivation wurde sogar eine Entlohnung von 5-10 Fl. für die Auslieferung des Täters auferlegt; diesen Betrag hatte der Täter später selbst zu entrichten. Die Strafe wurde ihm aufgrund der Straßenpolizeiordnung, ggf. nach dem II. Teil des Strafgesetzbuches bemessen. Hatte der Täter kein Geld zur Begleichung des Schadens, hatte er die Strafe auf der Straße abzuarbeiten, wobei der Tageslohn 5 Fl. betrug. Falls sein körperlicher Zustand keine physische Arbeit ermöglichte, wurde er mit Gefängnis bestraft. Mit der Hauptaufsicht über den Schutz von Bäumen wurde das Straßenpersonal beauftragt, dessen Aussage als ein voller Beweis galt. Die oben erwähnten Entlohnungen sollten auch andere Personen zur Einbeziehung in den Baumschutz motivieren.

Genauso wie Bäume, wurden auch die Straßenverkehrszeichen längs der Straßen auf Kosten des Grundbesitzers gebaut. Die oben angeführte Verordnung legte die Pflicht fest, die Kreuzungen an den Landeswegen mit Pappeln, ggf. mit anderen schnell anwachsenden den klimatischen Bedingungen entsprechenden Bäumen anzupflanzen und einen Wegweiser an diese anzubringen. Die Anpflanzung von Bäumen lag im Zuständigkeitsbereich einzelner Dominien und mit dem Unterhalt wurden die entsprechenden Gemeinden beauftragt.

\section{Die Zersplitterung der Rechtsregelung (zweite Hälfte des 19. - erste Hälfte des 20. Jahrhunderts)}

Die oben beschriebenen Regeln galten auf dem böhmischen Gebiet. Obwohl die Errichtung von Alleen zu den ganzstaatlichen Interessen gehörte, wurde dieser Bereich im 19. Jahrhundert nur aufgrund der Landesvorschriften geregelt. Aus dieser Sicht ist es nötig sich der Tatsache bewusst zu werden, dass drei unterschiedliche Rechtsregelungen (für Böhmen, Mähren und Schlesien) auf dem Gebiet der heutigen Tschechischen Republik galten. Seit der zweiten Hälfte des 19. Jahrhunderts entstanden neben den staatlichen ärarischen Straßen auch

13 Ca. zehn Jahre später stellt die Gub. Verordnung vom 26. Oktober 1837, G. Z. 31626 fest, dass viele Baumschulen entstanden. Dieser Vorschrift setzte dann weitere Anforderungen fest; diese Gärtchen sollten nur dem Unterricht dienen und es war verboten, dort Gemüse anzubauen. 
die nichtärarischen, deren Entstehung mit der Entwicklung der Selbstverwaltung zusammenhing. In einigen Fällen kam es zu einer unterschiedlichen Regulation für beide Straßenarten. Außerdem wurde die verfolgte Problematik auch thematisch aufgeteilt. Aus dem Erwähnten ergibt sich, dass die Rechtsregelung der Alleen längs der Straßen auf dem Gebiet der böhmischen Länder ziemlich zersplittert war.

Das Gesetz vom 2.12.1884 Böhm. L. G. Bl. Nr. 72, betreffend die Anpflanzung und den Schutz von Baumalleen oder Baumreihen längst der öffentlichen Straßen ${ }^{14}$ wurde zur Rechtsgrundlage der Regulierung von Alleen in Böhmen für die Zeit der folgenden mehr als 70 Jahre. In Schlesien galt das Gesetz vom 28.04.1896 Sles. L. G. Bl. Nr. 34, betreffend die Anpflanzung und den Schutz von Baumalleen oder Baumreihen längst der Reichs-, Bezirks- und Gemeindestraßen. In Mähren blieben die Allerhöchsten Reskripte vom 21.03.1740, 29.08.1752 und 24.09.1763 weiterhin in Kraft. Ungeachtet dessen waren einige Bestimmungen betreffend die Alleen auch im Gesetz vom 30.09.1877 Mähr. L. G. Bl., betreffend die Herstellung und Erhaltung der öffentlichen nichtärarischen Straßen und Wege enthalten.

$\mathrm{Zu}$ weiterer Gruppe die Alleen betreffenden Vorschriften gehörten die Anordnungen der Straßenpolizei; diese wurden wiederum für jedes Land separat erlassen. In Mähren und Schlesien betrafen sie lediglich nichtstaatliche (d.h. nichtärarische) Straßen und hatten Form eines Gesetzes. Für ärarische Straßen galt in Böhmen das Gesetz vom 15.04.1866 Böhm. L. G. Bl. Nr. 47, in Mähren das Gesetz vom 31.12.1874 Mähr. L. G. Bl., Nr. 5. Die Regelung der nichtärarischen Straßen richtete sich nach der Kundmachung des Statthalters vom 21. Juni 1886 Böhm. L. G. Bl. Nr. 54 (eine provisorische Straßenpolizeiordnung gültig für die Reichsstraßen im Königreiche Böhmen), in Mähren nach der Kundmachung des Statthalters vom 4.03.1892 Mähr. L. G. Bl. Nr. 29. In Schlesien wurde eine ähnliche Ordnung am 1.01.1878 Sles. L. G. Bl. Nr. 5 für nichtstaatliche Straßen erlassen ${ }^{15}$.

Nach der Entstehung der Tschechoslowakei änderten sich die Verhältnisse nicht, denn das erste tschechoslowakische Gesetz (Nr. 11/1918 Slg.) übernahm das bisher geltende Recht. Angesichts der Tatsache, dass sich der neue selbständige tschechoslowakische Staat auch aus der Slowakei und der Karpathoukraine zusammensetzte, scheint es als notwendig, auch die Rechtsregelung in diesen zwei Ländern kurz zu charakterisieren. In diesen Gebieten wurde das

14 Diese Vorschrift galt sowohl für die ärarische, als auch für die nichtärarischen Straßen. Dazu vgl. Pace, A. (Hrsg.), Ernst Mayerhofer's Handbuch für den politischen Verwaltungsdienst in den im Reichsrathe vertretenen Königreichen und Ländern. V. Band. Wien: Manz'sche k.u.k. Hof-Verlagsund Universitäts-Buchhandlung, 1901, S. S. 546, Anmerkung 3.

15 Slovník veřejného práva československého (Schlagwort „Silnice a cesty veřejné", bearbeitet von Jiří Čistecký). IV. Band. Praha: Eurolex Bohemia, 2000 (Reprint), S. 221-222. 
ungarische Recht übernommen, das im Vergleich zu der österreichischen Regelung fortschrittlicher war. Die Rechtsregelung war hier weder territorial, noch thematisch zersplittert. Die Problematik des Straßenbaus, der Straßen verwaltung und -unterhaltung, die Frage der Straßenpolizei und der Maut wurde im gesetzlichen Artikel I/1890 für das ganze ungarische Gebiet einheitlich geregelt. Die Problematik der Alleen betraf jedoch auch $₫ 49$ des gesetzlichen Artikels XII/1894, über die Feldwirtschaft und Feldpolizei, bzw. die Bestimmung der Durchführungsvorschrift zu diesem gesetzlichen Artikel ${ }^{16}$. In der Zeit der Ersten Tschechoslowakischen Republik kam es zu keiner Vereinheitlichung der Rechtsregelung betreffend die Regulierung von Straßen und Alleen.

Im Allgemeinen kann also gesagt werden, dass die Alleen oder zumindest Baumreihen längs aller Straßen angepflanzt werden sollten, sofern es sich um keine Waldstrecken handelte. Die Vorschriften unterschieden weiter über die Anpflanzung von Bäumen auf dem Straßenbankett oder erst hinter dem Straßengraben. Die Gemeinde sollte sich um die Bäume auf dem Straßenbankett kümmern ${ }^{17}$, und ihr oblag auch der Erlös aus diesen Bäumen. Die Bäume hinter dem Straßengraben wurden auf den Grundstücken der Privateigentümer angepflanzt und sie wurden auch mit der Pflege beauftragt; ihnen gehörte auch der Nutzen. Wie schon oben angedeutet wurde, waren sie über eine solche Pflicht überhaupt nicht begeistert, ungeachtet dessen konnte die Gemeinde sie dazu zwingen: einerseits konnte die Gemeinde den Grundstückseigentümern Geldstrafen auferlegen, andererseits konnte sie die Bäume auf deren Grundstücken selbst anpflanzen lassen. Auch in diesem Falle stand der Nutzen von Bäumen den Grundstückseigentümern $\mathrm{zu}^{18}$.

Die Baumpflege bestand vor allem im Schutz vor schädlichen Insekten (s. z.B. die Regierungsverordnung Nr. 58/1926 Slg., über die Bekämpfung von Maikäfern $)^{19}$. Das Beschneiden von Bäumen war nur ausnahmsweise zulässig - z.B. im Falle, dass diese den Verkehr verletzten. Weitere Ausnahmen wurden für die Telegrafenleitung (Gesetz Nr. 33/1922 Slg., und Regierungsverordnung Nr. 21/1923 Slg.) und für die elektrische Leitung (Gesetz Nr. 438/1919 Slg.) festgesetzt.

16 Es handelte sich um $\$ \$ 46$ und 49 der Verordnung des ehemaligen ungarischen Landwirtschaftsministeriums vom 17. 5. 1894 Nr. 48000; veröffentlicht In: Sbírka zákonů, nařízení a předpisů pro státní službu stavební v republice Československé, Band. III.).

17 Wie schon oben angeführt wurde, am Anfang des 19. Jahrhunderts sollten die Bäume erst hinter dem Straßengraben angepflanzt werden. Es ist also die Frage, was führte dazu, dass sie jetzt auf dem Straßenbankett angepflanzt wurden? Es kann sein, dass das die Folge einer Verbreiterung der Straßen war.

18 Slovník veřejného práva československého (Schlagwort „Silnice a cesty veřejné“, bearbeitet von Jiří Čistecký). IV. Band. Praha: Eurolex Bohemia, 2000 (Reprint), S. 221-222. 


\section{Die Rechtsregelung seit der zweiten Hälfte des 20. Jahrhunderts bis zur Gegenwart}

Die kommunistische Machtergreifung von 1948 bedeutete selbstverständlich eine radikale Veränderung für die ganze bisherige Rechtsordnung. Trotz sämtlicher Nachteile ist eine positive Tatsache zu erwähnen, und zwar, dass eine Rechtsvereinheitlichung durchgeführt wurde.

Die erste Vorschrift über die Rechtsregelung von Alleen nach 1948 stellte das Gesetz Nr. 147/1949 Slg., über die Erlassung einiger Straßenvorschriften dar. Dieses Gesetz erwähnt die Alleen jedoch nur im $₫ 2$ Abs. 2, nach dem „das Straßengehölz einen Bestandteil der Straßen bildet”. Auch das sog. Kommassationsgesetz (GesetzNr.47/1948Slg., über die technisch-wirtschaftliche Geländegestaltung) war von Bedeutung. In diesem Zusammenhang ist auch die Verordnung des Ministers für Technik Nr. 2/1950 Slg., über die Kompetenz der Nationalausschüsse bei Bau, Verwaltung und Unterhaltung von Staatsstraßen $\mathrm{zu}$ erwähnen. Diese Vorschrift übertrug die Straßenbäumepflege auf die Nationalausschüsse ( $\$ 1$ Abs. 3 Buchs. c). Wie es sich aus der zeitgenössischen Literatur ergibt ${ }^{20}$, blieben im Beobachtungszeitraum einige Prinzipien aus den oben erwähnten Vorschriften weiterhin in Kraft; in Mähren stammten sie sogar aus der Mitte des 18. Jahrhunderts. Erst in den folgenden Jahren wurden sie schrittweise ersetzt. Eine grundlegende Rolle spielten dabei die Empfehlungen des Rates des Kreisnationalausschusses ${ }^{21}$. Es ist nötig, sich der Tatsache bewusst $\mathrm{zu}$ werden, dass in dieser Zeit auch die politischen Texte einen gewissen gesetzgebenden Charakter aufwiesen.

$\mathrm{Zu}$ einer bedeutenden Änderung kam es im Rahmen der Kodifikationswelle in den sechziger Jahren, als das Gesetz Nr. 135/1961 Slg., über die Straßenverkehrswege (Straßengesetz) erlassen wurde. Dieses Gesetz wurde erst in den neunziger Jahren ersetzt (durch das bis heute geltende Gesetz Nr. 13/1997 Slg., über die Straßenverkehrswege). Das Gesetz Nr. 135/1961 Slg. erwähnte die Alleen im $\$ 14$; es bezeichnete die Straßen als „Straßengehölz”. Unter dem Begriff "Straßengehölz” verstand man sowohl Bäume, als auch Sträucher, wobei diese Bestimmung die Pflege auf die Bezirksnationalausschüsse übertrug. Eine ausführlichere Regelung wurde den Durchführungsverordnungen überlassen ${ }^{22}$.

Sollten wir an dieser Stelle die Grundunterschiede charakterisieren, dann ist vor allem die Tatsache zu erwähnen, dass die Anpflanzung von Bäumen

19 Im Allgemeinen handelte es sich um Durchführungsvorschriften zum Gesetz Nr. 165/1924 Slg., über den Schutz des Ackerbaus.

20 V. Vaníček, Silniční sadovnictví, Praha: Vědecko-technické nakladatelství, 1951, S. 6.

21 Ibidem, S. 7.

22 Zuerst Nr. 136/1961 Slg., später Nr. 35/1984 Slg. 
vereinheitlicht wurde und die Bäume von zuständigen Staatsorganen angepflanzt wurden. Ihnen oblagen auch die Pflege und der in Betracht kommende Erlös. Übrigens sollten die Bäume zwar nur hinter dem Straßengraben und nicht auf dem Straßenbankett angepflanzt werden ${ }^{23}$, jedoch kam es bei der Grundzusammenlegung zur Bildung eines Manipulationsstreifens zur Straßenunterhaltung. Gerade dieser Streifen wurde zur Anpflanzung von Bäumen ausgenutzt.

Vor allem in den fünfziger und sechziger Jahren ist eine Präferenz von Obstbäumen sowohl in der Literatur, als auch in den Rechtsvorschriften ersichtlich. Wenn auch Stimmen gegen das aus der Umgebung der Straßen stammende Obst auftraten, die Voraussetzung eines Wirtschaftsertrages für die Volkswirtschaft war lockend ${ }^{24}$. Bei der Anpflanzung wurden jedoch auch andere Aspekte berücksichtigt, als nur der Bodencharakter, wie z.B. der Einfluss der voreherrschenden Winde auf das Klima oder die konkreten Baumsorten ${ }^{25}$.

Es scheint, dass es in der zweiten Hälfte des 20. Jahrhunderts zu einer Änderung in der Konzeption der Vorschriften gekommen ist. Während der Schwerpunkt in der Regelung für die Anpflanzung von Bäumen in der Verordnung von 1961 liegt, macht die Verordnung aus den achtziger Jahren zuerst darauf aufmerksam, dass die Bäume den Straßenverkehr nicht stören dürfen. Trotzdem ist es nötig zu erwähnen, dass zu dieser Zeit mit den Alleen als einem festen Bestandteil der Verkehrsverbindungen gerechnet wurde $^{26}$. Was die Bäume auf dem Straßenbankett anbelangt, sollten sie bis 31.12.1990 beseitigt werden. Solche Bäume wurden nämlich für ein festes Verkehrshindernis gehalten ${ }^{27}$. Am 1.01 .1998 begann ein neues Gesetz Nr. 13/1997 Slg., über die Straßenverkehrswege zu gelten. Auch dieses Gesetz lässt den Straßenverkehrsbehörden einen freien Raum, ob sie die Beseitigung des Baumes beantragen - auch wenn er sich hinter dem Straßengraben befindet. Der Straßenverwalter ist verpflichtet, seine Absicht über das Fällen von Bäumen dem Organ des Naturschutzes bekannt zu geben. Dieses Organ kann diese Absicht ablehnen. Die Begründung der Absicht, Bäume fällen zu lassen, stützt sich vor allem auf $₫ 15$ des Gesetzes Nr. 13/1997 Slg.: „die Straßenvegetation... darf die Sicherheit der Benutzung des Verkehrsweges nicht

$23 \$ 19$ Abs.1 der Verordnung des Verkehrsministeriums Nr. 136/1961 Slg., über die Durchführung des Straßengesetzes; vgl. dazu V. Vaníček, Silniční sadovnictví, Praha: Vědecko-technické nakladatelství, 1951, S. 7.

24 V. Vaníček, Silniční sadovnictví, Praha: Vědecko-technické nakladatelství, 1951, S. 5.

25 Ibidem, S. 3-5.

26 „Bei den neuen Autobahnen, Straßen und Gemeindestraßen müssen die Anpflanzung und Art von Grünanlagen immer ein Bestandteil des Baus sein". $\$ 18$ Abs. 4 der Verordnung des Föderalverkehrsministeriums Nr. 35/1984 Slg.

27 Die Frist zur Beseitigung geht vom $₫ 21$ der Verordnung Nr. 35/1984 Slg. aus. 
bedrohen oder die Benutzung dieser Grundstücke zum Zwecke der Unterhaltung dieser Verkehrswege oder die Bewirtschaftung von benachbarten Grundstücken nicht disproportional erschweren". Hinter dieser nur sehr unklaren Bestimmung versteckt sich nur eine Bemühung des Eigentümers oder des Verwalters des Verkehrsweges, sich einer Einpflanzungspflicht und der Alleenpflege zu entbinden.

\section{Zum Schluss}

Die Beförderungskultur hat sich seit Schaffung der alten Baumalleen stark verändert. Das Reisen hat sich beschleunigt, wurde bequemer und der Verkehr wurde dichter. In den Autos leiden wir nicht mehr unter dem Wetter. Wir sind geschützt vor Sonne, Wind und Hitze. Die Funktionen der Bäume an den Alleen scheinen dem Autofahrer nicht mehr nötig. Sein Wunsch gilt vielmehr der schnelleren Fahrt. Doch entsprechen die alten Verkehrsverbindungen nicht mehr dem gegenwärtigen Verkehrsaufkommen. Die Straßen sind sehr schmal und die Bäume entlang der Straßen verhindern deren Ausbau. Auch erfordert schnelles Fahren schnelle Reaktionen und braucht ein übersichtliches Terrain, das die dichten Baumalleen nicht gewähren.

Darüber hinaus bedeutet eine Kollision mit einem Baum in hoher Geschwindigkeit praktisch den sicheren Tod $^{28}$. Das alles sind Faktoren, die dazu geführt haben, dass eine Reihe von Alleen längs der Straßen zugrunde gegangen is $\mathrm{t}^{29}$. In zahlreichen Fällen wird über die Zweckmäßigkeit von Baumfällungen in den tschechischen Medien berichtet ${ }^{30}$.

Auch das oft erwähnte Sicherheitskriterium ist nicht ganz eindeutig. In Österreich wurde eine Überwachung einer Straße vor und nach der Abholzung durchgeführt, wobei sich die Unfallzahl nach der Abholzung um 35\% erhöhte. Der Grund lag in der hohen Geschwindigkeit. Die Experten sind zu der Ansicht gekommen, dass die Bäume längst der Straßen als ein Maßstab eigener Geschwindigkeit und der Geschwindigkeit anderer Fahrzeuge dienen. Ohne diesen Maßstab fahren die Fahrer zu schnell ${ }^{31}$. Diese Ansicht bestätigt auch die Äußerung des Bürgermeisters der Gemeinde Pohled: „es ist wahr, dass seitdem

28 http://aktualne.centrum.cz/domaci/soudy-a-pravo/clanek.phtml?id=350718, zitiert am 6. September 2007, 12 Uhr.

$29 \mathrm{http} / /$ nasestromy.cz/modules.php?name=Web_Links\&l_op=viewlink\&cid=2, zitiert am 6. September 2007, 12 Uhr.

30 Marný boj o lípy v Neratovicích, http://www.cepo.arnika.org/kauza.shtml? x=1914665, 6. September 2007, 12 Uhr.

31 http://nasestromy.cz/obrazky/ln_1204.pdf, zitiert am 5. September 2007, 10 Uhr. 
hier die Bäume nicht mehr sind, fahren die Leute hier wie närrisch. Sie glauben, jetzt könne ihnen nichts passieren"32.

Die Bäume haben tatsächlich eine Bedeutung auch aus der Sicht der Verkehrssicherheit. Die Baumreihen zerstören den monotonen Charakter der Landschaft und zwingen damit die Fahrer zu einer größeren Vorsicht. Dank ihrer Größe dienen die Bäume als ein fahrendes Element, denn sie sagen den Fahrern vor, in welche Richtung die Straße hinter dem Horizont fortsetzt ${ }^{33}$.

In den letzten Jahren wurden die Alleen zum Bestandteil der tschechischen Landschaft. Bedeutend sind sie nicht nur aus der ökologischen, kultur-historischen oder landschaftsbildenden Sicht, sondern erhalten auch Bedeutung aus der Sicht des Straßenverkehrs. Man kann nur hoffen, dass die Alleen aus der Umgebung tschechischer Wege nicht verschwinden. Andererseits sind die Veränderungen im Straßenverkehr in Betracht zu nehmen und diesen Veränderungen auch die Anpflanzung von Alleen anzupassen ${ }^{34}$.

\section{Bibliography}

Handbuch aller unter der Regierung des Kaisers Joseph des II. Für die K.K. Erbländer ergangenen Verordnungen und Gesetze in einer Systematischen Verbindung 1780-1784. Band 3, Wien 1785.

Pace, A. (Hrsg.), Ernst Mayerhofer's Handbuch für den politischen Verwaltungsdienst in der Reichsrathe vertretenen Königreichen und Länder. V. Band. Wien: Manz'sche k.u.k. Hof-Verlags- und Universitäts-Buchhandlung, 1901.

Provinzialgesetzsammlung des Königreichs Böhmen für das Jahr 1828.

Provinzialgesetzsammlung des Königreichs Böhmen für das Jahr 1829.

Provinzialgesetzsammlung des Königreichs Böhmen für das Jahr 1831.

Provinzialgesetzsammlung des Königreichs Böhmen für das Jahr 1833.

Sammlung aller k. k. Verordnungen und Gesetze vom Jahre 1740 bis 1780, die unter Regierung des Kaisers Joseph des II. theils nochganz bestehen, theils zum Theile abgeändert sind, als ein Hilfs- und Ergänzungsbuch zu dem Handbuche aller unter der Regierung des Kaisers Joseph des II. für die k. k. Erbländer ergangenen Verordnungen und Gesetze in einer chronologischen Ordnung. IV. Band. Wien: Joh. Georg Mössle k.k. prov. Buchhändler, 1786.

32 V. Ševela, Stačí zpomalit, Respekt, 26. Ferbuar 2007. http://nasestromy.cz/obrazky/resp.pdf, zitiert am 2. řijna 2007, $14 \mathrm{Uhr}$.

33 http://www.mvcr.cz/casopisy/s/2007/10/konz.html, zitiert am 21. September 2007, 15 Uhr.

34 M. Míček, Názory: K čemu máme aleje? http://nasestromy.cz/modules.php?name=News\&file=cate gories\&op=newindex\&catid=4, zitiert am 7. September 2007, $13 \mathrm{Uhr}$. 
Sbírka zákonů, nařízení a předpisů pro státní službu stavební v republice Československé, Band. III.

Slovník veřejného práva československého (Schlagwort „Silnice a cesty veřejné“, bearbeitet von: Jiří Čistecký). IV. Band. Praha: Eurolex Bohemia, 2000 (Reprint).

Ševela, V., Stačí zpomalit. In: Respekt, 26. Februar 2007.

Vademecum pomocných věd historických. Praha: Svoboda, 1988

Vaníček, V., Silniční sadovnictví. Praha: Vědecko-technické nakladatelství, 1951

\section{WWW-Seiten}

http://aktualne.centrum.cz/domaci/soudy-a-pravo/clanek.phtml?id=350718, zitiert am 6. September 2007, $12 \mathrm{Uhr}$

http://nasestromy.cz/modules.php?name=Web_Links\&l_op=viewlink\&cid=2, zitiert am 6. September 2007, 12 Uhr.

http://nasestromy.cz/obrazky/ln_1204.pdf, zitiert am 5. September 2007, 10 Uhr.

http://www.mvcr.cz/casopisy/s/2007/10/konz.html, zitiert am 21. September 2007, 15 Uhr.

Marný boj o lípy v Neratovicích, http://www.cepo.arnika.org/kauza.shtml?x=1914665, 6. September 2007, 12 Uhr.

Míček, M. Názory: K čemu máme aleje?

http://nasestromy.cz/modules.php?name=News\&file=categories\&op=newindex\&catid=4, zitiert am 7. September 2007, 13 Uhr.

Vovesný, K, Tůmová, M., Kašperské Hory: stoletá alej musela ustoupit rekonstrukci silnice. http://www.ct24.cz/regiony/index_view.php?id=206883, zitiert am 2. Oktober 2007, 15 Uhr 